\title{
Relationship between pelagic larval duration and geographic distribution of Mediterranean littoral fishes
}

\author{
E. Macpherson ${ }^{1, *}$, N. Raventos ${ }^{1,2}$ \\ ${ }^{1}$ Centro de Estudios Avanzados de Blanes (CSIC), c/ Accés a la Cala St. Francesc 14, 17300 Blanes, Girona, Spain \\ ${ }^{2}$ Present address: École Pratique des Hautes Etudes, CNRS, UMR 8046, Université de Perpignan, 66860 Perpignan Cedex, France
}

\begin{abstract}
We examined the relationship between pelagic larval duration (PLD) - a predictor of a species' dispersal potential-and the geographic distribution range of 62 Mediterranean littoral fishes. We found a significant, positive, weak relationship between PLD and distribution range. This relationship was observed in species with long PLDs that can cross the few dispersal barriers (Macaronesian Islands) present in the Mediterranean, and in endemic Mediterranean species with short PLDs. Species with inshore larvae exhibited a shorter PLD than species with offshore larvae. Species with larvae living in spring-summer had shorter PLDs than those developing in autumn-winter. Mean geographic range was clearly smaller for species with inshore larval distributions than for species with offshore larval distributions. However, the geographic range of species with benthic eggs was smaller than that of pelagic spawners. The size of the distribution range of fishes is probably not controlled only by the PLD. The inshore/offshore position and the season of planktonic life play an important role in ensuring the return of larvae to their settlement habitats. Consequently, these factors also affect the size of the species' distribution range.
\end{abstract}

KEY WORDS: Early life history $\cdot$ Geographic range size $\cdot$ Pelagic larval duration $\cdot$ Mediterranean fishes $\cdot$ Biogeography $\cdot$ Dispersal

Resale or republication not permitted without written consent of the publisher

\section{INTRODUCTION}

The range of a species' geographic distribution and its dispersal capabilities are still central issues in marine ecology and biogeography. Recent studies have emphasized the importance of this relationship (Muss et al. 2001, Heads 2005). Most studies have revealed that it is difficult to apply common rules. However, several authors have shown that, in general, species with high dispersal capabilities have a larger geographic range than those with low dispersal potential (Kinlan \& Gaines 2003).

Pelagic larval duration (PLD) in fishes is considered a predictor of a species' dispersal potential. However, the existence of numerous exceptions in the dispersalrange size relationship indicates that additional aspects of early life fish biology should be researched (Armsworth et al. 2001, Shanks et al. 2003). Daily in- cremental marks on otoliths can be used to determine the duration of the larval phase. This provides a method for evaluating the relationship between PLD and a species' geographic distribution (Victor \& Wellington 2000, Lester \& Ruttenberg 2005). Despite several studies, no clear patterns have emerged to explain the relationship between these 2 factors. Some studies demonstrated a positive relationship between PLD and range (Zapata \& Herron 2002, Lester \& Ruttenberg 2005). In contrast, other studies found no significant relationship (Wellington \& Victor 1989, Victor $\&$ Wellington 2000). Other reports have demonstrated a positive relationship between gene flow and PLD, suggesting that larval strategies may be effective predictors of population genetic structure (Planes 2002).

Studies relating PLD and distribution range have used tropical reef fishes; see review by Lester \& Ruttenberg (2005). These authors suggested that the posi- 
tive relationship between these 2 variables in the IndoPacific can only be found in species with long PLDs that can cross significant dispersal barriers. These barriers are numerous in tropical reef systems, particularly in the Indo-Pacific and East Pacific, where the habitat is distributed among islands. Some of these islands are isolated by thousands of kilometres between patches of reef habitats. The habitat distribution in the Indo-Pacific and East Pacific tropical reef systems is quite different from that in temperate areas (e.g. the Mediterranean Sea). Therefore, it would be interesting to assess whether the patterns observed in tropical reef fishes can be generalized to other areas. The Mediterranean and eastern temperate Atlantic is characterised by a continuous coastline around the perimeter. There are a few oceanic islands (the Macaronesian Islands: Azores, Madeira and the Canaries), and few significant open-ocean barriers to dispersal (Macpherson 2002). Hence, we would expect to find no relationship between PLDs and range size distributions.

Dispersal potential can also be influenced by mechanisms other than PLD, such as inshore or offshore larval distributions and benthic or pelagic spawning strategies. Several studies document stronger transport currents offshore than inshore (Tintore et al. 1995, Largier 2003). Thus, larvae situated near the coastline would have lower dispersal possibilities than those situated along the continental shelf and slope (Shanks \& Eckert 2005). Furthermore, other studies show that larvae from benthic eggs are larger, better swimmers, and have more developed sensory systems than larvae from pelagic spawners (Blaxter 1986). The combination of these characteristics may make retention more likely for larvae from benthic spawners than for larvae from pelagic eggs, thereby affecting their dispersal capabilities (Hickford \& Schiel 2003). However, these aspects have not been considered in the context of the relationship between PLD and range size, with the exception of a recent study on California Current fishes (Shanks \& Eckert 2005). These authors suggested the existence of strong selective pressure to facilitate larval return to adult habitats, thus closing the larval pelagic phase. They called this problem the 'drift paradox'. They also suggested that selective pressure to solve this problem may involve the adaptation of early life traits to local oceanography, influencing the geographic range distribution of the species.

The Mediterranean Sea is inhabited by some 300 littoral fish species. Nearly 100 of these normally dwell at depths $<50 \mathrm{~m}$. There is a high proportion of endemic species (ca. $25 \%$ ) (Macpherson 2002). The geographic distribution and reproductive patterns of these species are well known (Whitehead et al. 1986) and, in addition, much is known about the planktonic larval distri- butions of numerous species (Sabatés 1990, Sabatés et al. 2003, A. Sabatés pers. comm.).

The western Mediterranean is characterized by a narrow continental shelf (100 $\mathrm{m}$ isobath, usually situated no more than $10 \mathrm{~km}$ from the coastline) and a permanent shelf/slope frontal system parallel to the bathymetry of the shelf. This front separates highsalinity open ocean water (greater than 38) from lowsalinity coastal water, partially as a consequence of continental discharges. The front runs along the continental slope and extends to a depth of some $400 \mathrm{~m}$, and the associated geostrophic current flow is $10 \mathrm{~km}$ wide at the surface, parallel to the front on its coastal side. The dynamics of the front displays a mesoscale activity, with formation of filaments, eddies and oscillations. The front has been found to delimit shelf and oceanic fish larvae and act as a barrier preventing dispersal of fish larvae out to the open sea (see Sabatés \& Olivar 1996, Sabatés et al. 2003 and references therein). The coastal area is also strongly influenced by seasonal changes in the predominant air masses. The predominant air mass blowing over the western Mediterranean basin is a dry, continental wind (Astraldi \& Gasparini 1992). During winter, strong winds are more frequent and can persist for several days, inducing intense water mixing and changes in the direction of flow, and bringing nutrients to the surface. In summer the pattern is very different, with weak onshore or shoreward winds.

In this study, the relationship between PLD and geographic range distribution of 62 littoral fishes was examined. We compared whether patterns observed in Mediterranean littoral fishes are similar to those observed in tropical reef fishes (Lester \& Ruttenberg 2005 and references therein). We also extended the analysis of Shanks \& Eckert (2005) by investigating whether combinations of early life traits (inshore/offshore distributions of larvae, spawning strategies and length of planktonic life) are related to local oceanography. Such a relationship would facilitate the closure of the larval pelagic phase and influence the geographic distribution of species.

\section{MATERIALS AND METHODS}

Sampling was performed along the coast off Blanes, Spain (ca. $42^{\circ} 02^{\prime} \mathrm{N}, 3^{\circ} 13^{\prime} \mathrm{E}$, north-west Mediterranean) between spring 2001 and autumn 2004. Additional samples were taken in the western Mediterranean Sea, from the north-east of Spain to the Straits of Gibraltar. Following the methodology described in Raventos \& Macpherson (2001), a minimum of 3 samples were taken weekly during the settlement period for most of the species (spring-summer). One or 2 samples were 
taken weekly in autumn-winter (Garcia-Rubiés \& Macpherson 1995, Raventos \& Macpherson 2001, 2005, Macpherson \& Raventos 2005, authors' unpubl. data for settlement period identification). Samples were collected at depths of between 0 and $20 \mathrm{~m}$ (occasionally as far down as $30 \mathrm{~m}$ ) by SCUBA divers using hand nets. New settlers were identified according to different authors and by personal observations made in previous studies (see Raventos \& Macpherson 2001 and references therein). Fish were measured in the laboratory within $1 \mathrm{~h}$ of capture. Otoliths (sagittae and lapilli) were removed and preserved in $96 \%$ ethanol. The size of newly settled individuals ranged from 4 to $20 \mathrm{~mm}$, depending on the species (see Raventos \& Macpherson 2001). In all cases, the size of post-settlers was under $40 \mathrm{~mm}$ at no more than $30 \mathrm{~d}$ after settlement.

PLDs were estimated using the daily otolith increment technique. Otoliths (lapillus and sagitta) were mounted on microscope slides using Eukitt as the mounting medium. After mounting, otoliths were polished to expose all the daily increments within the same plane. Readings were made using a light microscope connected to a digital camera and an image analysis system. PLD was defined as the number of increments occurring from the centre of the otolith outwards to the settlement mark. All individuals examined had a settlement mark (see Wilson \& McCormick 1999, Raventos \& Macpherson 2001 for settlement mark identifications). Sub-daily increments (i.e. faint increments occurring between dark, well-defined increments) were found on the otoliths of some species. However, we followed the criteria used by Wellington \& Victor (1989) and Raventos \& Macpherson (2001) for interpreting which increments constitute daily growth increments. As previous authors have done (e.g. Wellington \& Victor 1989), we assumed that increments formed during the larval stage represented daily increments. However, as Brothers et al. (1983) noted, the initiation time for daily increments in our study species was not known. Thus, our counts could slightly over- or underestimate the length of larval life, depending on the exact timing of the growth-increment initiation. For some species (Symphodus roissali, S. tinca, Chromis chromis and Lipophrys trigloides), we observed that increment deposition in laboratory-reared larvae started on the first day of hatching (Macpherson \& Raventos 2005, Raventos \& Macpherson 2005).

PLD was estimated from 2389 otoliths from 59 littoral species. We also considered data obtained in previous papers (Vigliola 1998, Raventos \& Macpherson 2001). In all, we examined 62 species (3046 otoliths) belonging to 13 families (see Table 1).

For each species we examined: mean, minimum and maximum PLD; the spawning strategy (benthic or pelagic broadcast spawners); larval distribution (inshore or offshore waters); the season of pelagic larval life; and the geographic distribution range (Table 1). We collected spawning type and geographic range size information for each species from Whitehead et al. (1986) and the FishBase data bank (www.fishbase.org/ home.htm). The larval distributions of both inshore (<100 m depth) and offshore (>100 m depth) plankton were collected from different studies covering the coastal area and continental shelf and slope of the north-west Mediterranean. Such studies were carried out over several years, and included Sabatés (1990) and Sabatés et al. (2003) and references therein (additional information was obtained from A. Sabatés pers. comm.). The distinction between inshore and offshore zones was made by differences in physical oceanography between the 2 habitats, and was based primarily on the distance from the shore. Inshore waters are dominated by waves and bottom stress and have slower alongshore flows (Largier 2003). Shanks \& Eckert (2005) used the inshore area (<30 m depth) and shelf/slope ( $>30 \mathrm{~m}$ depth) to separate species by adult habitat. These authors used the $30 \mathrm{~m}$ isobath because each group of fishes released larvae into water with different oceanographic conditions. However, in the present study, as a narrow coastal shelf characterizes the study area, we used a more conservative distance (the location of the $100 \mathrm{~m}$ isobath) for larval distribution. The season of each species' larval pelagic life was estimated using the PLD and settlement period (see Garcia-Rubies \& Macpherson 1995, Raventos \& Macpherson 2001, and present data). Most species only spawn in one season. However, for those species that spawn in 2 seasons (e.g. Lipophrys trigloides in late autumn and winter; Sarpa salpa in spring and autumn) we considered only the season in which most larvae were found (Garcia-Rubies \& Macpherson 1995, Macpherson \& Raventos 2005).

Distribution range size was calculated following the examples of previous studies (Victor \& Wellington 2000, Lester \& Ruttenberg 2005). We estimated 3 measures of range size: latitudinal range, longitudinal range, and maximum linear surface distance $(\mathrm{km})$ of the furthest 2 points of the known species range. The maximum linear distance was determined from the surface of the globe and not from a flat projection. Adult mobility might influence range distribution independently of early life characteristics. However, most species have littoral benthic habitats and probably do not cross large coastal discontinuities (e.g. between the continent and islands). Only atherinids show pelagic behaviour, mostly along the littoral zone.

We regressed all range size measurements against PLD. In all analyses, measurements were log-transformed to attain normality. Analyses were conducted: (1) using all data; and (2) by limiting analyses to sam- 
Table 1. Pelagic larval duration (mean, SD, maximum and minimum) in days, and maximum geographic range of littoral species in the Mediterranean Sea; $\mathrm{N}=$ total no. of ind., $\mathrm{Np}=$ no. of ind. from previous papers (Vigliola 1998 [ ${ }^{*}$ ] and Raventos \& Macpherson 2001). Distribution of each species in biogeographic provinces is listed. NECS = Northeast Atlantic Continental Shelf $\left(50-65^{\circ} \mathrm{N}\right), \mathrm{CNRY}=$ Canary Current Coastal $\left(20-50^{\circ} \mathrm{N}\right)$, GUIN $=$ Guinea Current Coastal $\left(15^{\circ} \mathrm{S}-20^{\circ} \mathrm{N}\right), \mathrm{MAC}=\mathrm{Macaronesian}$ Islands $\left(25-40^{\circ} \mathrm{N}\right.$; see Macpherson 2002); I = inshore ( $<1$ mile) larval distribution, $\mathrm{O}=$ offshore ( $>1$ mile) larval distribution, $\mathrm{B}=$ benthic eggs, $\mathrm{P}=$ pelagic eggs; season of planktonic life: $\mathrm{W}=$ winter, $\mathrm{S}=$ spring, $\mathrm{SU}=$ summer, $\mathrm{A}=$ autumn

\begin{tabular}{|c|c|c|c|c|c|c|c|c|c|c|c|c|c|c|}
\hline Species & $\mathrm{N}$ & Mean & $\mathrm{SD}$ & Min. & Max. & $\mathrm{Np}$ & $\begin{array}{l}\text { Larval } \\
\text { dist. }\end{array}$ & Eggs & Season & NECS & CNRY & GUIN & MAC & $\begin{array}{l}\text { Range } \\
(\mathrm{km})\end{array}$ \\
\hline \multicolumn{15}{|l|}{ Apogonidae } \\
\hline Apogon imberbis & 63 & 21.3 & 1.4 & 18 & 24 & 8 & & B & SU & & + & + & + & 5480 \\
\hline \multicolumn{15}{|l|}{ Atherinidae } \\
\hline Atherina boyeri & 72 & 10.4 & 0.9 & 9 & 12 & 17 & I & B & SU & & + & & + & 4805 \\
\hline Atherina hepsetus & 83 & 11.4 & 1.3 & 9 & 14 & 24 & I & $\mathrm{B}$ & SU & & + & & + & 4384 \\
\hline \multicolumn{15}{|l|}{ Bleniidae } \\
\hline Aidablennius sphynx & 61 & 41.2 & 2.5 & 35 & 45 & 11 & $\mathrm{O}$ & B & SU & & + & & & 3625 \\
\hline Coryphoblennius galerita & 30 & 25.8 & 1.1 & 24 & 28 & 2 & $\mathrm{O}$ & $\mathrm{B}$ & $\mathrm{S}$ & & + & & + & 5480 \\
\hline Lipophrys adriaticus & 5 & 23.0 & 1.7 & 21 & 24 & 2 & & $\mathrm{~B}$ & SU & & & & & 2951 \\
\hline Lipophrys canevai & 24 & 30.7 & 1.0 & 28 & 34 & 3 & $\mathrm{O}$ & $\mathrm{B}$ & SU & & & & & 3625 \\
\hline Lipophrys trigloides & 299 & 67.2 & 10.7 & 39 & 89 & 15 & & $\mathrm{~B}$ & W & & + & & + & 4384 \\
\hline Parablennius incognitus & 54 & 23.8 & 1.6 & 21 & 30 & 10 & & $\mathrm{~B}$ & SU & & + & & + & 4384 \\
\hline Parablennius pilicornis & 2 & 28 & - & 28 & 28 & & $\mathrm{O}$ & B & $\mathrm{S}$ & & + & + & & 7778 \\
\hline Parablennius sanguinolentus & 2 & 33 & 1.4 & 32 & 34 & 1 & $\mathrm{I}$ & $\mathrm{B}$ & $\mathrm{S}$ & & + & + & & 4667 \\
\hline Parablennius zvonimiri & 2 & 24 & - & 24 & 24 & & & $\mathrm{~B}$ & SU & & & & & 2951 \\
\hline \multicolumn{15}{|l|}{ Bothidae } \\
\hline Arnoglossus thori & 14 & 31.4 & 3.7 & 25 & 39 & & $\mathrm{O}$ & $\mathrm{P}$ & $\mathrm{A}$ & & + & & & 3778 \\
\hline Bothus podas & 41 & 33.7 & 2.6 & 28 & 38 & & $\mathrm{O}$ & $\mathrm{P}$ & $\mathrm{A}$ & & + & + & & 3889 \\
\hline \multicolumn{15}{|l|}{ Clinidae } \\
\hline Clinitrachus argentatus & 53 & 26.2 & 1.1 & 23 & 29 & 4 & & B & $\mathrm{S}$ & & & & & 3625 \\
\hline \multicolumn{15}{|l|}{ Gadidae } \\
\hline $\begin{array}{l}\text { Gaidropsarus meditterraneus } \\
\text { Gobiesocidae }\end{array}$ & 1 & 43 & - & 43 & 43 & 1 & $\mathrm{O}$ & $\mathrm{P}$ & $\mathrm{W}$ & + & + & & & 4047 \\
\hline Apletodon dentatus & 21 & 14.4 & 0.7 & 13 & 15 & 3 & I & B & $\mathrm{S}$ & & + & & & 2361 \\
\hline Apletodon incognitus & 20 & 14.1 & 1.1 & 12 & 15 & & I & $\mathrm{B}$ & SU & & & & & 2361 \\
\hline Diplecogaster bimaculata & 11 & 11.8 & 1.1 & 10 & 14 & & I & $\mathrm{B}$ & SU & + & + & & & 3334 \\
\hline Gouania wildenowi & 23 & 13.1 & 1.6 & 11 & 17 & 1 & I & B & SU & & & & & 2951 \\
\hline Lepadogaster candollei & 12 & 13.2 & 0.9 & 12 & 15 & 1 & $\mathrm{I}$ & $\mathrm{B}$ & SU & + & + & & + & 3445 \\
\hline \multicolumn{15}{|l|}{ Gobiidae } \\
\hline Gobius bucchichi & 22 & 19.2 & 1.0 & 17 & 21 & 10 & & B & SU & & + & & & 4047 \\
\hline \multicolumn{15}{|l|}{ Labridae } \\
\hline Coris julis & 75 & 28.1 & 3.6 & 21 & 34 & 23 & $\mathrm{O}$ & $\mathrm{P}$ & SU & + & + & + & + & 7223 \\
\hline Ctenolabrus rupestris & 66 & 20.9 & 2.0 & 18 & 28 & 12 & $\mathrm{I}$ & $\mathrm{P}$ & $\mathrm{S}$ & + & + & & & 4445 \\
\hline Labrus viridis & 22 & 31.3 & 2.5 & 27 & 35 & 1 & I & $\mathrm{B}$ & $\mathrm{S}$ & & + & & & 3625 \\
\hline Symphodus cinereus & 54 & 11.6 & 1.0 & 10 & 14 & 3 & I & B & SU & & + & & & 3625 \\
\hline Symphodus doderleini & 56 & 13.0 & 0.9 & 11 & 15 & 3 & I & $\mathrm{B}$ & SU & & & & & 3372 \\
\hline Symphodus mediterraneus & 72 & 13.6 & 1.1 & 11 & 16 & 9 & $\mathrm{I}$ & $\mathrm{B}$ & SU & & & & & 3625 \\
\hline Symphodus melops & 2 & 15.0 & 0.0 & 15 & 15 & 2 & $\mathrm{I}$ & $\mathrm{B}$ & $\mathrm{S}$ & + & + & & + & 3334 \\
\hline Symphodus ocellatus & 220 & 10.1 & 1.1 & 8 & 13 & 23 & $\mathrm{I}$ & $\mathrm{B}$ & $\mathrm{S}$ & & & & & 3372 \\
\hline Symphodus roissali & 292 & 12.6 & 1.6 & 9 & 18 & 106 & I & $\mathrm{B}$ & $\mathrm{S}$ & & & & & 3625 \\
\hline Symphodus rostratus & 10 & 14.8 & 1.6 & 13 & 17 & 2 & I & B & $\mathrm{S}$ & & & & & 3372 \\
\hline Symphodus tinca & 82 & 10.8 & 1.0 & 9 & 13 & 4 & I & B & $\mathrm{S}$ & & & & & 3625 \\
\hline Thalassoma pavo & 71 & 38.9 & 5.2 & 30 & 48 & 11 & $\mathrm{O}$ & $\mathrm{P}$ & SU & & + & & + & 5480 \\
\hline Xyrichtis novacula & 33 & 34.2 & 6.0 & 28 & 55 & 1 & & $\mathrm{P}$ & A & & + & + & & 4445 \\
\hline \multicolumn{15}{|l|}{ Mullidae } \\
\hline Mullus surmuletus & 52 & 30.1 & 2.1 & 28 & 35 & & $\mathrm{O}$ & $\mathrm{P}$ & $\mathrm{S}$ & & + & & & 4047 \\
\hline \multicolumn{15}{|l|}{ Pomacentridae } \\
\hline Chromis chromis & 425 & 18.6 & 1.6 & 15 & 25 & 17 & $\mathrm{O}$ & B & SU & & + & + & & 5556 \\
\hline \multicolumn{15}{|l|}{ Sciaenidae } \\
\hline Sciaena umbra & 3 & 22.5 & 0.7 & 22 & 23 & & & $\mathrm{P}$ & $\mathrm{S}$ & & + & & + & 4890 \\
\hline \multicolumn{15}{|l|}{ Scorpaenidae } \\
\hline Scorpaena porcus & 1 & 29 & - & 29 & 29 & 1 & $\mathrm{O}$ & $\mathrm{P}$ & $\mathrm{S}$ & & + & & + & 5901 \\
\hline Scorpaena scofra & 1 & 25 & - & 25 & 25 & & $\mathrm{O}$ & $\mathrm{P}$ & $\mathrm{S}$ & & + & & + & 5901 \\
\hline
\end{tabular}


Table 1 (continued)

\begin{tabular}{|c|c|c|c|c|c|c|c|c|c|c|c|c|c|c|}
\hline Species & $\mathrm{N}$ & Mean & $\mathrm{SD}$ & Min. & Max. & $\mathrm{Np}$ & $\begin{array}{c}\text { Larval } \\
\text { dist. }\end{array}$ & Eggs & Season & NECS & CNRY & GUIN & MAC & $\begin{array}{c}\text { Range } \\
(\mathrm{km})\end{array}$ \\
\hline \multicolumn{15}{|l|}{ Serranidae } \\
\hline Epinephelus marginatus & 21 & 24.6 & 1.3 & 22 & 30 & & $\mathrm{O}$ & $\mathrm{P}$ & SU & & + & + & + & 10001 \\
\hline Serranus cabrilla & 54 & 24.3 & 1.8 & 21 & 28 & 3 & $\mathrm{O}$ & $\mathrm{P}$ & $\mathrm{S}$ & & + & + & + & 9445 \\
\hline Serranus hepatus & 22 & 18.0 & 0.9 & 16 & 22 & & $\mathrm{O}$ & $\mathrm{P}$ & SU & & + & & + & 2223 \\
\hline Serranus scriba & 12 & 28.2 & 1.6 & 26 & 32 & & $\mathrm{O}$ & $\mathrm{P}$ & S & & + & + & + & 9445 \\
\hline \multicolumn{15}{|l|}{ Sparidae } \\
\hline Boops boops & 23 & 16.9 & 0.9 & 16 & 20 & 14 & $\mathrm{O}$ & $\mathrm{P}$ & SU & + & + & + & + & 6112 \\
\hline Diplodus annularis & 74 & 18.3 & 1.4 & 16 & 21 & 15 & $\mathrm{O}$ & $\mathrm{P}$ & SU & & + & & + & 4468 \\
\hline Diplodus cervinus & 3 & 18.3 & 1.5 & 17 & 20 & 1 & $\mathrm{O}$ & $\mathrm{P}$ & $\mathrm{S}$ & & + & & + & 4890 \\
\hline Diplodus puntazzo & 80 & 32.7 & 4.8 & 19 & 48 & $80^{*}$ & $\mathrm{O}$ & $\mathrm{P}$ & $\mathrm{A}$ & & + & + & + & 4805 \\
\hline Diplodus sargus & 80 & 27.6 & 4.3 & 19 & 37 & $80^{*}$ & $\mathrm{O}$ & $\mathrm{P}$ & $\mathrm{S}$ & & & & & 4047 \\
\hline Diplodus vulgaris & 80 & 40.8 & 5.7 & 29 & 58 & $80^{*}$ & $\mathrm{O}$ & $\mathrm{P}$ & $\mathrm{W}$ & & + & + & + & 5889 \\
\hline Oblada melanura & 55 & 16.2 & 1.3 & 14 & 18 & 25 & $\mathrm{O}$ & $\mathrm{P}$ & SU & & + & + & + & 5556 \\
\hline Pagellus erythrinus & 10 & 43.9 & 2.8 & 40 & 49 & & $\mathrm{O}$ & $\mathrm{P}$ & $\mathrm{S}$ & + & + & & + & 4384 \\
\hline Pagrus pagrus & 1 & 38 & - & 38 & 38 & 1 & $\mathrm{O}$ & $\mathrm{P}$ & $\mathrm{S}$ & & + & & + & 4384 \\
\hline Sarpa salpa & 74 & 31.7 & 2.5 & 27 & 38 & 11 & $\mathrm{O}$ & $\mathrm{P}$ & W & & + & & + & 4778 \\
\hline Spondyliosoma cantharus & 12 & 33.7 & 2.9 & 29 & 38 & 2 & $\mathrm{O}$ & $\mathrm{B}$ & $\mathrm{S}$ & + & + & + & + & 7556 \\
\hline \multicolumn{15}{|l|}{ Syngnathidae } \\
\hline Nerophis ophidion & 2 & 21.5 & 0.7 & 21 & 22 & & I & B & $\mathrm{S}$ & + & + & & & 4047 \\
\hline Syngnathus typhle & 2 & 15.0 & 0.0 & 15 & 15 & & $\mathrm{I}$ & $\mathrm{B}$ & $\mathrm{S}$ & + & + & & & 4047 \\
\hline \multicolumn{15}{|l|}{ Trachinidae } \\
\hline Trachinus draco & 10 & 12.9 & 0.7 & 12 & 14 & & $\mathrm{O}$ & $\mathrm{P}$ & SU & + & + & & + & 4805 \\
\hline \multicolumn{15}{|l|}{ Triglidae } \\
\hline Trigla lucerna & 12 & 19.1 & 1.2 & 18 & 21 & & $\mathrm{O}$ & $\mathrm{P}$ & $\mathrm{W}$ & + & + & & & 5001 \\
\hline \multicolumn{15}{|l|}{ Tripterygiidae } \\
\hline Trypterygion delaisi & 25 & 17.3 & 1.1 & 16 & 21 & 3 & I & B & $\mathrm{S}$ & & + & & & 3625 \\
\hline Trypterygion melanurus & 53 & 17.6 & 2.3 & 15 & 25 & 6 & I & B & $\mathrm{S}$ & & & & & 3625 \\
\hline Trypterygion tripteronutus & 39 & 18.4 & 2.8 & 16 & 27 & 5 & I & B & S & & & & & 3625 \\
\hline
\end{tabular}

ples that comprised over 20 ind. per species (Victor \& Wellington 2000, Lester \& Ruttenberg 2005). A Bonferroni correction was used to correct for multiple comparisons.

PLD differences among species with different spawning strategies (benthic and pelagic spawners), larval distributions (inshore and offshore), and seasons of planktonic life were tested using ANOVA. We also compared differences between Medi-terranean endemic and non-endemic species, and between species that have colonized oceanic islands (the Macaronesian Islands: Azores, Madeira and Canaries) and those that are only distributed along the continental coast. These islands are situated at ca. 1880, 760 and $100 \mathrm{~km}$ from the nearest continental coast, respectively. Mediterranean endemic species are defined as those living in the Mediterranean and waters around the Straits of Gibraltar (Gulf of Cadiz and north-west Morocco). We used the full dataset (using counts of individual otoliths), but only included species with more than 5 individuals. We ran these tests as nested ANOVAs, nesting species within type (e.g. inshore or offshore larvae, benthic or pelagic spawner) as a random factor. A Bonferroni correction was used to account for multiple tests of these analyses.
We also tested whether larval distributions and spawning characteristics of the species were related to their distribution range size. Pairwise comparisons ( $t$ tests) were used to compare maximum geographic range sizes of species with inshore vs. offshore larvae, and benthic vs. pelagic eggs.

\section{RESULTS}

The life traits of the 62 species (Table 1 ) showed that 35 species produce demersal eggs and 27 are pelagic spawners; 23 species were identified to have inshore larvae, whereas 30 species were identified to have offshore larvae (data were not available for 9 species). Most species with inshore larvae (92\%) were benthic spawners. The larvae of most species had their planktonic period in spring (28 species) and summer (25 species). Four species had their planktonic period in autumn, and 5 in winter. Eighteen species were endemic to the Mediterranean, and 28 species can be found in the Macaronesian Islands.

Correlation results showed that all 3 geographic range measurements were highly significant: $\mathrm{r}=0.89$ and 0.83 $(p<0.0001)$ for maximum linear distance vs. longitudinal 
and latitudinal range, respectively; $0.62(p<0.0001)$ for longitudinal vs. latitudinal range. Therefore, in accordance with Lester \& Ruttenberg (2005), we used maximum linear distance as the measure of a species' geographic extent. Correlations between mean PLD vs. maximum and minimum PLD were also highly co-linear (0.94 and 0.89, $\mathrm{p}<0.0001$, respectively). We used mean PLD as the measure of a species' pelagic larval duration (Victor \& Wellington 2000).

The relationship between PLD and geographic ranges was positive across species (and thus significant $; r=0.30, p<0.01$ ). However, this only explained a low proportion of variance (Fig. 1). When analyses were limited to samples comprised of over 20 ind. per species, the relationship was positive but non-significant (Table 2). However, values differed between families; they were highly positive and significant for Labridae $(r=0.65, p=0.01)$ and non-significant for Blenniidae and Sparidae. A significantly positive relationship was also observed in those species with benthic spawning strategies $(r=0.36, p=0.003$; Table 2). The other relationships were non-significant after Bonferroni correction. We considered these 2 relationships (spawning strategies and larval distributions) separately. However, benthic spawners have predominantly inshore larvae, and pelagic spawners mostly offshore larvae (Table 1).

Species with inshore larvae exhibited a shorter PLD $($ mean $=13.4 \mathrm{~d})$ than species with offshore larvae $($ mean $=26.3 \mathrm{~d}$, ANOVA, $\mathrm{p}<0.001)$. Furthermore, mean PLD was shorter for benthic spawners than for

Table 2. Correlation analyses of PLDs and distribution ranges, using maximum linear distance $(\mathrm{km})$. Analyses include all species (left columns) and those with sample sizes $>20$ ind. (right columns). $\mathrm{N}=$ number of species, $\mathrm{ns}=$ not significant ( $p$-values calculated after Bonferroni correction)

\begin{tabular}{|c|c|c|c|c|c|c|}
\hline & $\begin{array}{l}\mathrm{N} \\
\text { All }\end{array}$ & $\mathrm{R}$ & $\mathrm{p}$ & $\begin{array}{c}\mathrm{N} \\
>20 \text { in }\end{array}$ & d. ${ }^{\mathrm{R}}$ & $\mathrm{p}$ \\
\hline All species & 62 & 0.30 & 0.01 & 38 & 0.27 & ns \\
\hline \multicolumn{7}{|l|}{ Families } \\
\hline Blenniidae & 9 & 0.02 & ns & 5 & 0.28 & $\mathrm{~ns}$ \\
\hline Labridae & 13 & 0.65 & 0.01 & 11 & 0.64 & 0.03 \\
\hline Sparidae & 11 & -0.06 & ns & 7 & -0.16 & ns \\
\hline \multicolumn{7}{|c|}{ Ecological characteristics } \\
\hline Inshore larvae & 23 & 0.23 & ns & 16 & 0.28 & $\mathrm{~ns}$ \\
\hline Offshore larvae & 29 & -0.02 & $\mathrm{~ns}$ & 16 & -0.04 & $\mathrm{~ns}$ \\
\hline Benthic eggs & 35 & 0.36 & 0.003 & 20 & 0.43 & $\mathrm{~ns}$ \\
\hline Pelagic eggs & 27 & -0.03 & ns & 18 & -0.03 & $\mathrm{~ns}$ \\
\hline Endemics & 18 & 0.26 & $\mathrm{~ns}$ & 15 & 0.40 & $\mathrm{~ns}$ \\
\hline Non-endemics & 44 & 0.24 & ns & 23 & 0.13 & $\mathrm{~ns}$ \\
\hline $\begin{array}{l}\text { Macaronesian } \\
\text { colonizers }\end{array}$ & 38 & 0.25 & ns & 17 & 0.01 & ns \\
\hline Non-colonizers & 24 & 0.29 & ns & 21 & 0.37 & ns \\
\hline
\end{tabular}

pelagic spawners. PLD was also shorter for Mediterranean endemic species than for non-endemics. However, the results were not significant after Bonferroni correction (Table 3). The species that have colonized oceanic islands (the Macaronesian Islands: Azores,
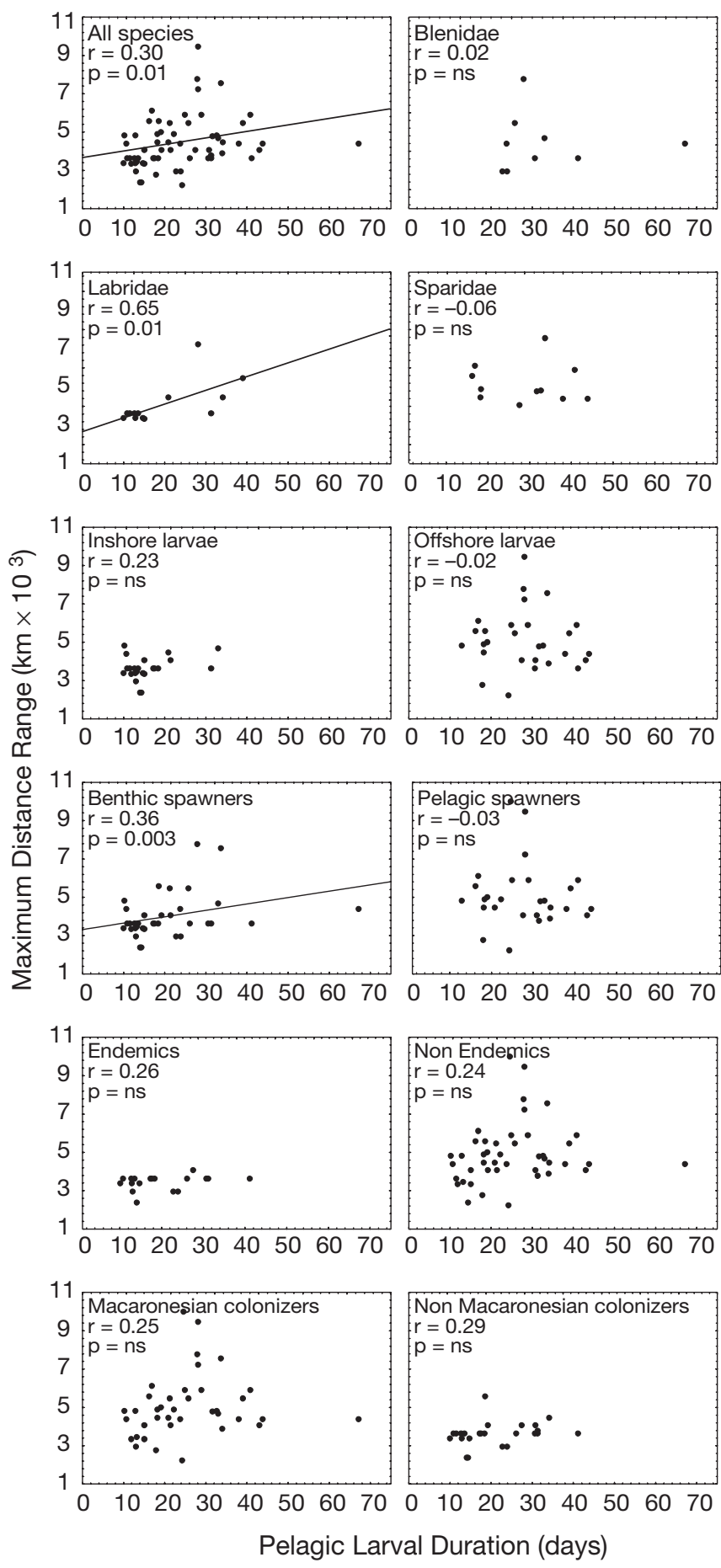

Fig. 1. Relationships between mean planktonic larval duration (PLD) and maximum geographic range for different groups of species. $\mathrm{ns}=$ not significant 
Table 3. PLD differences among species, taking into account ecological distribution characteristics. Values are results of nested ANOVAs using the full dataset (counts of individual otoliths) excluding species with $<5$ ind. Species within type (e.g. inshore or offshore, benthic or pelagic eggs) are used as a random factor. $\mathrm{N}=$ number of species, $\mathrm{NO}=$ number of otoliths, $a, b, c=$ post hoc Newman-Keuls test results ( $\mathrm{p}$-values calculated after Bonferroni correction)

\begin{tabular}{|lrrrrrrrr|}
\hline Group & N & NO & $\begin{array}{c}\text { Mean } \\
\text { PLD }\end{array}$ & SD & MS & $F$ & $\mathrm{p}$ \\
& & & & & & \\
\hline Inshore larvae & 19 & 1150 & 13.4 & 4.12 & 8046.1 & 13.08 & $<0.001$ \\
Offshore larvae & 23 & 1388 & 26.3 & 8.87 & & & \\
Benthic eggs & 30 & 2135 & 23.9 & 19.1 & 30146.3 & 0.44 & 0.509 \\
Pelagic eggs & 22 & 959 & 28.4 & 8.5 & & & \\
Mediterranean & 18 & 1140 & 16.7 & 8.6 & & & \\
$\quad$ endemics & & & & & 38251.2 & 3.49 & 0.067 \\
Non-endemics & 34 & 1954 & 30.3 & 18.1 & & & \\
Macaronesian & 28 & 1356 & 34.7 & 19.9 & & & \\
$\quad$ Is. colonizers & & & & & 32429.9 & 6.62 & 0.013 \\
Non-colonizers & 24 & 1738 & 17.9 & 8.0 & & & \\
Winter $(a)$ & 4 & 465 & 55.8 & 18.8 & & & \\
Spring $(b)$ & 19 & 1174 & 18.8 & 9.3 & 15408.2 & 11.68 & $<0.001$ \\
Summer $(b)$ & 25 & 1287 & 19.2 & 6.9 & & & \\
Autumn $(c)$ & 4 & 168 & 33.2 & 4.6 & & & $a>c>b$ \\
& & & & & & & \\
\end{tabular}

\section{DISCUSSION}

We observed a weak, positive relationship between PLD (as a proxy of potential dispersal) and distribution range of Mediterranean littoral fishes. Species with a long PLD tended to have a larger geographic range than those with a short PLD. This supports the results of some studies on tropical reef fishes (e.g. Zapata \& Heron 2002, Lester \& Ruttenberg 2005). However, the low proportion of variance accounted for by this relationship suggests that PLD is not a strong predictor of the distribution range size. Other mechanisms may also influence the distribution patterns exhibited by fishes.

Lester \& Ruttenberg (2005) suggested that the positive relationship between PLD and range size in Indo-Pacific reef fishes is generated by species with long PLDs that can cross dispersal barriers. In our study, species whose ranges extend to oceanic islands (Azores, Madeira,

Madeira and the Canaries) had significantly longer PLDs than those living exclusively along the European and African coasts.

Species characterised by planktonic life in spring and summer showed a significantly shorter PLD (mean $=18.8$ and $19.2 \mathrm{~d}$, respectively) than those developing in winter $($ mean $=55.8 \mathrm{~d})$ and autumn $($ mean $=33.2 \mathrm{~d})$ (Table 3). All species found in winter and autumn (except Lipophrys trigloides), in periods with strong winds and changes in the direction of flow had an offshore distribution and pelagic eggs (Table 1).

Finally, mean geographic range was clearly smaller for species with inshore larval distributions than for species with offshore larval distributions ( $t$-test, $\mathrm{p}<$ 0.001). However, species with benthic eggs had range sizes smaller than those of pelagic spawners ( $t$-test, $\mathrm{p}<0.001$; Table 4). To further explore the relationship among geographic range, larval distribution and spawning characteristics, we examined the relationship between residuals from regressions of PLD against range size. These residuals (factoring out PLD) were also significantly related (for inshore-offshore larvae, $t$-test: $t=-2.668$, $\mathrm{df}=50, \mathrm{p}=0.01$; for benthic-pelagic eggs: $t=-2.985$, df $=60, \mathrm{p} \leq 0.004)$. This further supports the notion that some factors that are correlated with spawning characteristics and larval position act as important controls on geographic range size.
Table 4. Pairwise comparisons (t-test) among maximum distribution ranges (Max. distr.; km) of species, taking into account their ecological characteristics. Analyses include all species (left columns) and those with sample sizes $>20$ ind. (right columns). $\mathrm{N}=$ number of species ( $\mathrm{p}$-values calculated after Bonferroni correction)

\begin{tabular}{|c|c|c|c|c|c|c|c|}
\hline Group & $\begin{array}{c}\mathrm{N} \\
\text { All }\end{array}$ & $\begin{array}{l}\text { Max. } \\
\text { Mean }\end{array}$ & $\begin{array}{r}\text { distr. } \\
\text { SD }\end{array}$ & $\mathrm{p}$ & $\begin{array}{c}\mathrm{N} \\
>20 \text { ind. }\end{array}$ & $\begin{array}{l}\text { Max. distr. } \\
\text { Mean SD }\end{array}$ & $\mathrm{p}$ \\
\hline $\begin{array}{l}\text { Inshore larvae } \\
\text { Offshore larvae }\end{array}$ & $\begin{array}{l}23 \\
29\end{array}$ & $\begin{array}{l}3679 \\
6104\end{array}$ & $\begin{array}{r}628 \\
2055\end{array}$ & $<0.001$ & $\begin{array}{l}16 \\
16\end{array}$ & $\begin{array}{rr}3641 & 688 \\
6345 & 2033\end{array}$ & $<0.001$ \\
\hline $\begin{array}{l}\text { Benthic eggs } \\
\text { Pelagic eggs }\end{array}$ & $\begin{array}{l}32 \\
30\end{array}$ & $\begin{array}{l}4058 \\
5964\end{array}$ & $\begin{array}{l}1511 \\
2008 a\end{array}$ & $<0.001$ & $\begin{array}{l}20 \\
18\end{array}$ & $\begin{array}{rr}3762 & 799 \\
6494 & 2070\end{array}$ & $<0.001$ \\
\hline
\end{tabular}


have PLDs that are significantly shorter (mean $=19.7 \mathrm{~d}$ ) than labrids from the eastern Pacific (mean $=46.6 \mathrm{~d}, t$ test, p < 0.0001) (Victor \& Wellington 2000). Furthermore, other Mediterranean endemics belong to taxa with PLDs that are shorter than 1 mo and that have inshore larvae (e.g. Gobiesocidae, Tripterygiidae). The PLDs of Mediterranean nearshore fishes are also shorter than those of other temperate nearshore fishes, e.g. California Current species (Shanks \& Eckert 2005).

The source of these differences is not easy to explain. However, the characteristics of the Mediterranean littoral fishes and their distribution patterns can be influenced by events that are not directly related to PLD, e.g. historical processes. Such processes (e.g. the closing and opening of the Mediterranean Sea and periodic glaciation events over the past 1 million yr) have favoured speciation events and the geographic distribution of numerous extant species in the North Atlantic (Mix et al. 2001). These historical processes have conditioned endemism, e.g. as exhibited by labrids (Hanel et al. 2002). They have also affected species flow, both between oceanic islands and mainland areas, and between Mediterranean and Atlantic basins (Bargelloni et al. 2003). The effects of these processes can be observed in the gene flow between fish populations from mainland areas and oceanic islands. Genetic studies have demonstrated that short-PLD species from oceanic islands (e.g. Tripterygion delaisi) were isolated by such climatic fluctuations (Carreras-Carbonell et al. 2005). Other species with longer PLDs could capitalize on an unobstructed gene flow between mainland areas and oceanic islands (Muss et al. 2001). Although genetic studies on this topic remain scarce, findings suggest that historical processes should be taken into account when comparing potential dispersal abilities and distribution ranges (Heads 2005).

The results obtained using residuals and factoring out PLD showed that range size distributions can be controlled by larval position and spawning strategies. Armsworth et al. (2001) showed that incorporation of larval biology and behavioural considerations into dispersal models markedly changes their predictions. Additionally, larvae residing near the bottom disperse short distances, thus affecting predictions from PLD (Shanks et al. 2003). The larval behaviour of Mediterranean species is mostly unknown, and further studies may clarify its influence on dispersal capabilities. Furthermore, species with larvae situated inshore showed shorter PLDs and smaller ranges than species with larvae situated offshore. Larvae from demersal eggs are usually more abundant inshore, whereas larvae from pelagic spawners are more common offshore (Sabatés 1990, Suthers \& Frank 1991, but see Hickford \& Schiel 2003). As different authors have pointed out (Blaxter 1986), pelagic eggs produce smaller larvae with less- developed sensory systems and poorer swimming abilities. Indeed, they usually require a longer planktonic period to reach settlement size. This combination of factors, together with stronger transport events offshore (Tintore et al. 1995), may make retention less likely for these larvae. This leads to higher dispersion capabilities and larger geographic range sizes.

The differences in life history traits of species that have planktonic larvae in spring-summer or in autumn-winter suggests that these species were well adapted to local oceanography, as indicated by Shanks \& Eckert (2005) for California Current species. Mediterranean species that have their planktonic life in autumn and winter had significantly longer PLDs than those developing in spring and summer. The spawning and planktonic life of most littoral species in the Mediterranean takes place in spring and summer, when water temperature and phyto- and zooplankton productivities are higher (Estrada et al. 1985). These environmental conditions are more favourable for larval survival. Furthermore, the wind regime in springsummer (low inshore winds) facilitates the closure of the pelagic phase of larvae with short PLDs. Larvae with PLDs shorter than 2 wk (most inshore larvae) would have great difficulties in surviving and returning to settlement habitats in offshore wind conditions (e.g. winter). Furthermore, wind conditions have significant effects on the spawning strategies of the species. The reproductive season of most benthic spawners is in late spring and summer, when strong winds are less frequent and their negative effects on nests lower (Raventos \& Macpherson 2005). As Shanks \& Eckert (2005) pointed out, the early life traits of the species may show an adaptation to the local oceanography to avoid alongshore loss of larvae, thus promoting settlement of larvae into their parental habitats. This trend was observed in Mediterranean littoral fishes in the present study, and suggests that the hypotheses made by Sinclair (1988) and Shanks \& Eckert (2005) for California Current species could be extrapolated to other areas.

In conclusion, PLD is not a strong predictor of the range size distribution of Mediterranean littoral fishes. The positive relationship between these 2 variables can be generated by: species with long PLDs, which can cross the few barriers to dispersal (Macaronesian Islands), and Mediterranean endemic species, with short PLDs. These results agree with those observed in the Indo-Pacific tropical reef system (Lester \& Ruttenberg 2005). However, the range size distribution of fishes is not only controlled by PLDs. The inshore/offshore position and season of planktonic life play an important role in ensuring the return of larvae to their settlement habitats. Consequently, these factors also affect a species' distribution range size. 
Acknowledgements. We thank A. L. Shanks, B. I. Ruttenberg and 2 anonymous reviewers for interesting comments and suggestions made on the first version of our manuscript. We also appreciate the comments of our colleagues, A. GarciaRubies, I. Uriz, M. Becerro and A. Sabatés, who helped in preparing and reviewing the manuscript. This study was supported by MCYT projects CTM2004-05265 and BOS200305904.

\section{LITERATURE CITED}

Armsworth PR, James MK, Bode L (2001) When to press on or turn back: dispersal strategies for reef fish larvae. Am Nat $157: 434-450$

Astraldi M, Gasparini GP (1992) The seasonal characteristics of the circulation in the North Mediterranean Basin and their relationship with the atmospheric-climatic conditions. J Geophys Res 97:9531-9540

Bargelloni L, Alarcon JA, Alvarez MC, Penzo E, Magoulas A, Reis C, Patarnello T (2003) Discord in the family Sparidae (Teleostei): divergent phylogeographical patterns across the Atlantic-Mediterranean divide. J Evol Biol 16: $1149-1158$

Blaxter JHS (1986) Development of sense organs and behavior of teleost larvae with special reference to feeding and predator avoidance. Trans Am Fish Soc 115:98-114

Brothers EB, Williams DM, Sale PF (1983) Length of larval life in twelve families of fishes at 'One Tree Lagoon', Great Barrier Reef, Australia. Mar Biol 76:319-324

Carreras-Carbonell J, Macpherson E, Pascual M (2005) Rapid radiation and cryptic speciation in Mediterranean triplefin blennies (Pisces: Tripterygiidae) combining multiple genes. Mol Phyl Evol 37:751-761

Estrada M, Vives F, Alcaraz M (1985) Life and productivity of the open sea. In : Margalef R (ed) Key environments. Western Mediterranean. Pergamon Press, Oxford, p 148-197

Garcia-Rubies A, Macpherson E (1995) Substrate use and temporal pattern of recruitment in juvenile fishes of the Mediterranean littoral. Mar Biol 124:35-42

Hanel R, Westneat MW, Strumbauer C (2002) Phylogenetic relationships, evolution of brood care behaviour, and geographic speciation in the wrasse tribe Labrini. J Mol Evol 55:776-789

Heads M (2005) Towards a panbiogeography of the seas. Biol J Linn Soc 84:675-723

Hickford MJH, Schiel DR (2003) Comparative dispersal of larvae from demersal versus pelagic spawning fishes. Mar Ecol Prog Ser 252:255-271

Kinlan BP, Gaines SD (2003) Propagule dispersal in marine and terrestrial environments: a community perspective. Ecology 84:2007-2020

Largier J (2003) Considerations in estimating larval dispersal distances from oceanographic data. Ecol Appl 13:S71-S89

Lester SE, Ruttenberg BI (2005) The relationship between pelagic larval duration and range size in tropical reef fishes: a synthetic analysis. Proc R Soc Lond B 272:585-591

Macpherson E (2002) Large scale gradients of species richness in the Atlantic Ocean. Proc R Soc Lond B 269: $1715-1720$

Macpherson E, Raventos N (2005) Temporal patterns of settlement and early life history traits in two temperate littoral fishes in the NW Mediterranean. Mar Biol 148:167-177
Mix AC, Bard E, Schneider R (2001) Environmental processes of the ice age: land, oceans, glaciers (EPILOG). Q Sci Rev 20:627-657

Muss A, Robertson DR, Stepien CA, Wirtz P, Bowen BW (2001) Phylogeography of Ophioblennius: the role of the ocean currents and geography in reef fish evolution. Evolution 55:561-572

Planes S (2002) Biogeography and larval dispersal inferred from population genetic analysis. In: PF Sale (ed) Coral reef fishes. Dynamics and diversity in a complex ecosystem. Academic Press, Amsterdam, p 201-220

Raventos N, Macpherson E (2001) Planktonic larval duration and settlement marks on the otoliths of Mediterranean littoral fishes. Mar Biol 138:1115-1120

Raventos N, Macpherson E (2005) Environmental influences on temporal patterns of settlement in two littoral labrid fishes in the Mediterranean Sea. Estuar Coast Shelf Sci 63: 479-487

Robertson DR (2001) Population maintenance among tropical reef fishes: inferences from small-island endemics. PNAS 98:5667-5670

Sabatés A (1990) Distribution pattern of larval fish populations in the northwestern Mediterranean. Mar Ecol Prog Ser 59:75-82

Sabatés A, Olivar MP (1996) Variation of larval fish distributions associated with variability in the location of a shelfslope front. Mar Ecol Prog Ser 135:11-20

Sabatés A, Zabala M, Garcia-Rubies A (2003) Larval fish communities in the Medes Islands marine reserve (north-west Mediterranean). J Plankton Res 25:1035-1046

Shanks AL, Eckert GL (2005) Population persistence of California Current fishes and benthic crustaceans: a marine drift paradox. Ecol Monogr 75:505-524

Shanks AL, Grantham BA, Carr MH (2003) Propagule dispersal distance and the size and spacing of marine reserves. Ecol Appl 13:S159-S169

Sinclair M (1988) Marine populations: an essay on population regulation and speciation. Washington Sea Grant Program, Seattle, WA

Suthers IM, Frank KT (1991) Comparative persistence of marine fish larvae from pelagic versus demersal eggs off southwestern Nova-Scotia, Canada. Mar Biol 108: $175-184$

Tintore J, Wang DP, Garcia E, Viudez A (1995) Near-inertial motion in the coastal ocean. J Mar Syst 6:301-312

Victor BC, Wellington GM (2000) Endemism and the pelagic larval duration of reef fishes in the eastern Pacific Ocean. Mar Ecol Prog Ser 205:241-248

Vigliola L (1998) Contrôle et régulation du recruitment des sparidae (Poissons, Téléostéens) en Méditerranée: importance des processus pré- et post-installation benthique. $\mathrm{PhD}$ thesis, University Aix-Marseille II

Wellington GM, Victor BC (1989) Planktonic larval duration of one hundred species of Pacific and Atlantic damselfishes (Pomacentridae). Mar Biol 101:557-567

Whitehead PJP, Bauchot ML, Hureau JC, Nielsen J, Tortonese E (1986) Fishes of the Northeastern Atlantic and the Mediterranean. UNESCO, Paris

Wilson DT, McCormick MI (1999) Microstructure of settlement-marks in the otoliths of tropical reef fishes. Mar Biol 134:29-41

Zapata FA, Herron PA (2002) Pelagic larval duration and geographic distribution of tropical eastern Pacific snappers (Pisces: Lutjanidae). Mar Ecol Prog Ser 230:295-300

Submitted: November 24, 2005; Accepted: May 3, 2006

Proofs received from author(s): November 7, 2006 